\title{
Les mutineries dans les manuels scolaires français de l'entre-deux-guerres aux années 1980
}

\author{
Emmanuelle Picard
}

Chargée de recherche au Service d'histoire de l'éducation, INRP-ENS

Les mutineries de 1917 sont, dès l'origine, un enjeu de débat dans l'espace public, dont les modalités évoluent entre la fin de la guerre et aujourd'hui. En tant que tel, elles invitent à s'interroger sur le traitement qui fut le leur dans la catégorie bien particulière de l'espace éditorial que constituent les manuels scolaires. Dans ce domaine, les choix dont elles font l'objet entretiennent sans surprise des liens avec deux problématiques distinctes : les mutineries en tant qu'objet éditorial (sujet de témoignages publiés, de documents, d'essais ou de travaux universitaires) et la morphologie des programmes scolaires en général. Les évolutions de ces deux objets étant différenciées, elles induisent des effets spécifiques sur leur traitement dans la production à usage pédagogique.

Les mutineries n'ont été que très récemment l'objet de travaux universitaires, si l'on exclut le premier d'entre eux, la thèse de Guy Pédroncini publiée en 1967, qui est aussi l'un des tous premiers travaux universitaires portant sur un quelconque aspect de la Première Guerre mondiale. Dès lors, la question des sources utilisées par les auteurs des manuels n'est pas sans poser problème car, si l'on excepte l'ouvrage de Pierre Renouvin publié en 1934 [Renouvin, 1934], les études relatives à la guerre ne sont, pendant la majeure partie de la période étudiée ici, pas le fait d'universitaires mais de témoins ou de politiques [Olivera, 2001]. Il y a donc à s'interroger sur les conditions intellectuelles du discours sur les mutins dans l'espace public et leurs retranscriptions dans les ouvrages scolaires. Par ailleurs, les programmes scolaires imposent des cadres narratifs spécifiques, dans lesquels s'insèrent les manuels. Il sera donc nécessaire de s'interroger sur les effets qu'ils auront dans les choix concernant la mention des 
mutineries, ne serait-ce qu'en raison des découpages chronologiques qu'ils imposent ${ }^{1}$.

Pour conserver une cohérence à cette étude, les manuels considérés ici sont les seuls manuels de lycée, la Première guerre mondiale étant traitée en Terminale depuis les années 1920 jusqu'au début des années 1980, puis "rétrocédé » en classe de Première avec les nouveaux programmes de $1982^{2}$. Cette césure qui n'a pas, a priori, d'effet attendu sur le traitement de l'objet étudié ici, s'inscrit cependant dans une reconfiguration méthodologique de l'enseignement de l'histoire et dans un contexte de transformation de la production universitaire qui aura des effets sur le traitement général des mutineries de 1917 dans les manuels scolaires.

\section{De L'ENTRE-DEUX-GUERRES AUX ANNEES 1960 :}

\section{UN EVENEMENT EXCLUSIVEMENT MILITAIRE}

La question des mutineries est évoquée dans les manuels scolaires dès l'Entredeux-guerres car, en 1929, la Première Guerre mondiale fait son entrée dans les programmes d'enseignement d'histoire (nouveau programme de Philosophie et de Mathématiques : 1850-1920, décret de 1925) [Tison, 1994].

La plupart des manuels de cette époque y consacrent une cinquantaine de pages, dans lesquelles les opérations militaires sur tous les fronts sont extrêmement détaillées, cartes à l'appui. Elles représentent $90 \%$ du contenu relatif à la guerre, l'arrière étant évoqué de façon très succincte. En revanche, les portraits des chefs militaires sont abondants et les opérations stratégiques longuement décrites selon un récit organisé chronologiquement. L'année 1917 fait l'objet, comme avant elles les années 1914, 1915 et 1916, d'une partie spécifique, généralement décrite sous le vocable d' " année tournante » ou « année du tournant de la guerre ». La question de l'entrée en guerre

\footnotetext{
1 Sur l'ensemble de la période considérée, la proportion des pages consacrées à la Première guerre mondiale a nettement diminué : $12,4 \%$ entre 1920 et $1940 ; 7,1 \%$ entre 1945 et $1980 ; 7,8 \%$ entre 1980 et 1994 , alors même que les bornes de la période traitée dans le programme se resserrait [Tison, 1995].

${ }^{2}$ Les manuels scolaires peuvent être identifiés grâce à la base de données EMMANUELLE mise en œuvre par Alain Choppin (Service d'histoire de l'éducation, INRP) et accessible en ligne : http://www.inrp.fr/she/choppin_emmanuelle.htm
} 
des Américains (et donc de la guerre sous-marine) et celle de la Révolution russe (et donc de la fermeture du front oriental) en constituent les morceaux essentiels (les trois quarts du texte). Elle est aussi l'occasion de présenter les « nouveaux chefs » chez les différents belligérants, signe de ce tournant évoqué. On peut ainsi lire dans le Delagrave de 1929 : «La guerre qui se prolonge amène en Allemagne et en France un changement dans le haut commandement, rendu responsable là de l'échec de Verdun, ici des hécatombes des offensives partielles. Or la guerre même a révélé des hommes. » [Calvet-Ancel, 1929]

La question des mutineries n'est évoquée qu'en fin de chapitre, généralement de façon concomitante avec la mention d'une crise qui touche les différentes armées. L'exemple français est cependant le seul à être développé et se trouve contextualisé dans une réflexion sur la conduite de la guerre, sans qu'aucune mention ne soit faite des mouvements à l'arrière. On est dans une logique de crise de longue durée, dont les mutineries dans l'armée française constituerait le dernier avatar avant le redressement durable de l'été 1917. Ce type d'analyse n'est pas nouveau et reprend l'un des poncifs des écrits sur la guerre publiés durant la période [Mermeix, 1919].

Voici le récit organisé de cette crise, tel que l'on peut le lire dans le Delagrave : « La crise commence par un conflit entre le GQG et les Chambres qui, après les pertes de la guerre d'usure et de Verdun, exigent un contrôle parlementaire aux armées général et permanent (juillet 1916) : leurs délégués, sans intervenir dans la conduite des opérations, s'occuperont du matériel, de la santé, du ravitaillement des troupes. Les lacunes de l'organisation défensive devant Verdun amènent, la bataille finie, le départ de Joffre (décembre 1916); l'échec meurtrier du vain essai de percée en Champagne (offensive du 16 avril 1917) cause le limogeage de son successeur incapable. A cette crise du commandement, se superpose une crise de l'armée: provoquées par les sacrifices inutiles, attaques mal préparées, fatigues, souffrances, permissions mal réglées, éclatent des mutineries, ou plus exactement des grèves : des régiments refusent de remonter aux tranchées (fin avril 1917). »

Cette analyse est en fait très étroitement liée à celle que l'on peut lire dans la production de l'époque traitant de la Grande Guerre. En effet, si les mutineries n'ont fait à l'époque l'objet d'aucune publication spécifique [Offenstadt, 2005], elles sont 
mentionnées dans les différentes histoires de la guerre qui sont alors publiées, et généralement traitées comme le résultat de la conjonction entre une armée épuisée et un choix stratégique, et/ou politique (l'arrête de l'offensive) préjudiciables [Hanotaux, 1923 ; Painlevé, 1919]. Elles sont généralement minimisées, présentées comme une crise paradoxalement à la fois très sérieuse et très limitée, et sont davantage l'occasion de s'arrêter sur la reprise en main par Pétain que sur les causes profondes du mouvement. On retrouve cette tendance dans les manuels scolaires qui ne leur consacrent que quelques lignes allusives, quant ils font une place plus consistance à la personnalité et à l'action de Pétain. On observe un mode narratif dominant qui fait une place centrale aux rôles des personnalités militaires : les mutineries sont l'occasion de souligner et d'exposer l'action « efficace» du général Pétain. En revanche, l'idée d'un mouvement soutenu et encouragé par les « pacifistes », «révolutionnaires » et autres « défaitistes », si présente dans les histoires de la Grande Guerre publiées à l'époque [Cazals, Rousseau, 2001] ne se retrouve pas dans les manuels, qui cantonne ce mouvement à l'armée.

Seul le Malet-Isaac de 1930 propose une vision plus générale de la crise. Celle-ci s'inscrit d'ailleurs dans des choix intellectuels qui rompent assez largement avec ceux des autres manuels. Si le Malet-Isaac contient comme tout un chacun de longs développement sur les conditions diplomatiques préalables à la guerre (sujet alors central de la réflexion sur la guerre) et le déroulement des opérations militaires, on y trouve aussi de substantiels paragraphes sur la vie dans les tranchées et les conditions de combat, avec (chose rare) des illustrations. Le récit étant également organisé de façon chronologique, l'entrée « mutinerie » se trouve donc dans le chapitre consacré à l'année 1917, qui est celle de «L'intervention des Etats-Unis » (titre du chapitre). Mais dès la première ligne, il est précisé que cette année, la plus troublée de la guerre, le fut davantage par les événements politiques et économiques que par les événements militaires. Le cours commence pourtant par la guerre sous-marine et l'entrée en guerre américaine, puis par la Révolution russe. Les offensives sur le front de l'ouest ne font l'objet que de quelques lignes lapidaires.

Puis vient le paragraphe final sous le titre, «Le malaise général » : « La guerre en se prolongeant créait partout un état de lassitude et de crise, que la révolution russe 
vint encore aggraver. (...) Le malaise général se manifesta par des troubles d'ordre politique, social et militaire, et par diverses tentatives de paix ». Il décrit en premier lieu la lassitude dans les empires centraux, puis en France : «beaucoup pensaient que la victoire était impossible et qu'une paix de compromis valait mieux que l'épuisement total : on les appela défaitistes. Après l'échec sanglant du 16 avril, le moral de l'armée fléchit; des mutineries éclatèrent. Mais cette crise de confiance ne dura pas : l'armée fut reprise en main par son nouveau chef, Pétain. » On peut rapprocher cette analyse de celle présentée par Pierre Renouvin dans son ouvrage en 1934 qui propose une analyse alliant la lassitude des combattants et les erreurs stratégiques, dans un contexte de contestation politique.

Reste que cette tentative de contextualisation est extrêmement superficielle et que les mutineries ne sont l'objet que de quelques lignes dans un ensemble par ailleurs conséquent.

Le contenu des programmes ne se modifie pas radicalement dans les années qui suivent la Seconde Guerre mondiale et les mutineries ne gagnent pas véritablement du terrain, alors même que le propos général se modifie graduellement. La Première Guerre mondiale n'est plus cantonnée désormais au seul récit des batailles, stratégies et vies des grands hommes. Elle accède progressivement au statut d'événement complet, impliquant des dimensions sociales et économiques (on parle désormais de trois thèmes : la guerre, les civils, l'industrie), dont la présence modifiera légèrement le traitement fait des mutins, sans pour autant que le choix d'une narration chronologique ne soit remis en question. Le manuel le plus «exemplaire» dans ce domaine est la version réactualisée du manuel de Jules Isaac, publié en 1953 chez Hachette: la narration linéaire de la guerre s'entrecroise de pages mettant en perspectives les armées en présence, détaillant la dimension industrielle du conflit, bien au-delà de ce qu'il faisait dans l'édition précédente. En revanche, le texte sur 1917 est le même au mot près.

Dans le Hatier de 1945 [Genet, 1945], l'année 1917 est introduite par le titre suivant : «Les mécomptes de l'année 1917 ». Qualifiée d'année « des déceptions », elle est encore l'occasion de longs développements sur la situation militaire des 
différents fronts. Dans la partie sur les mécomptes de l'Entente, l'offensive Nivelle est présentée rapidement, accompagnée d'un laconique « échec indiscutable », suivi d'une présentation tout aussi succincte de Caporetto. D'autres manuels consacrent encore une place substantielle à la description de l'offensive comme le Masson de 1946 [Hallynck, 1946] avec sa description minutieuse sur une page entière sans que les mutineries ne soient mentionnées. Ces dernières sont alors évoquées dans le bilan de l'année 1917 avec dorénavant l'introduction d'une problématique plus générale et surtout la mention du rôle potentiel d'exemple de la révolution en Russie. Pour autant, les mutineries restent encore traitées dans leur dimension purement militaire et ne sont que juxtaposées, matériellement, avec les événements russes. Dans le Hatier de 1945, «les déceptions de 1917 ébranlent le moral des combattants » et des signes de défaillance apparaissent. La Révolution russe est mentionnée, juste avant que ne soit développé l'exemple français : «Chose plus grave, le mouvement atteint l'armée. Des soldats refusent de monter en ligne, envoient des délégations à leurs chefs de corps pour dire qu'ils n'obéiront pas aux ordres d'attaque ; des émeutes éclatent à Soissons où deux régiments veulent marcher sur Paris. »

Le Masson de 1946 choisit un angle d'approche similaire avec son paragraphe consacré à «la crise du moral » qui commence par le remplacement de Nivelle. «L'échec d'une opération dont Nivelle avait garanti le succès produisit une très fâcheuse impression, tant dans les troupes que dans les milieux politiques et l'opinion publique. De nombreux députés, autorisés à suivre les péripéties de la bataille, ignorants des dures réalités de la guerre, exagérèrent les sacrifices subis par nos soldats et répandirent un vent de suspicion. Le gouvernement décida de relever Nivelle qui fut remplacé le 15 mai par le général Pétain. (...). » Le paragraphe suivant est directement consacré aux mutineries : «Sous l'exemple de la révolution russe, comme sous l'effet d'une active propagande pacifiste auprès des permissionnaires et des unités cantonnées au repos à l'arrière, des mutineries graves se produisirent à partir du 20 mai dans les troupes. Des régiments, glorieux souvent, refusèrent de monter en ligne et certains tentèrent même de marcher sur Paris. » On voit alors apparaître l'idée d'une cause directement politique aux mutineries françaises.

Dans un manuel destiné à l'enseignement catholique [Aimond, 1949], le même 
système explicatif est repris et amplifié : la Révolution russe fait l'objet d'un traitement très rapide (une demi page) à laquelle succède une page et demi sur «l'échec des offensives alliées ». Elle est presque toute entière consacrée à une longue description des conditions de l'offensive du 16 avril, avec une minoration de son échec : «Il fallut arrêter l'offensive » le succès n'étant pas assez décisif et coûtant cher. Il s'ensuite partout en France, « une profonde déception ». "Le général Nivelle dût céder le commandement en chef au général Pétain, un autre vainqueur de Verdun », qui va alors s'atteler à relever le moral de l'armée «profondément atteint par l'échec de la grande offensive d'avril. D'ailleurs, des agents défaitistes travaillaient alors à soulever les troupes et même à les pousser contre Paris. »

Le seul manuel qui tranche véritablement avec cette approche très codifiée est celui publié sous la direction de C. Morazé et P. Wolff chez A. Colin. Le chapitre sur la victoire des alliés commence traditionnellement avec le renversement militaire de 1917, la défection des Russes et l'arrivée des Américains. La mention des mutineries est lapidaire : après une phrase sur l'inflation et les salaires, les privations et les deuils, une mention est faite de Zimmerwald, puis : «Dans tous les Etats, des crises trahissent la lassitude des peuples : en France, le pacifisme aboutit aux grèves et aux mutineries de troupes de 1917 ; l'Angleterre doit faire face à la révolte de Pâques 1916 en Irlande. » Guère de place non plus pour les mutins, même s'ils ne sont plus pour autant isolés dans leurs retranchements militaires mais s'inscrivent dans un mouvement de protestation plus général.

Sur l'ensemble de cette période, les mutineries ne sont donc pas passées sous silence et le terme même est généralement employé. Pour autant, elles sont cantonnées à une désobéissance militaire limitée, du simple fait de la place qui leur est accordée. Elles sont plus qu'un objet le prétexte pour une description assez précise de la politique menée par Pétain à partir de mai 1917 et mal reliées aux autres événements sociaux et politiques de la période.

La première révision d'ampleur des programmes d'histoire intervient à la fin des années 1950 avec un nouveau programme décidé en 1957 et applicable en 1962. Ce programme, dit de «civilisation», s'accompagne d'une réduction de la place de 
l'histoire dans la formation des lycéens. Le choix d'approche thématique restreint les longs développements consacrés aux combats et de fait, les mutineries disparaissent presque complètement des manuels. Le manuel publié en 1963 chez de Gigord en fait ainsi un traitement rapide [Roulié, 1963] : «Sur le front occidental, Pétain calma des mutineries qui avaient éclaté dans quelques régiments. » Les causes n'en sont pas précisées, mais le contexte général est rappelé : «Chez tous les belligérants, l'opinion publique commençait à manifester sa réprobation contre une guerre meurtrière et interminable. » La plupart du temps cependant, les manuels des années soixante se contentent de reprendre les mentions qu'ils en faisaient dans leurs éditions précédentes.

On voit cependant se mettre en place à cette époque une lecture plus politique des mutineries, qui les inscrit dans un contexte faisant la part belle aux agitateurs et autres agents allemands. Les mutins ne sont plus cantonnés dans le seul espace du rejet d'une offensive désastreuse pour accéder à celui d'un refus plus général. Le manuel publié aux Éditions de l’École en 1955 [Jarry, 1955] précise ainsi, après avoir mentionné l'agitation générale : «Certains milieux syndicaux extrêmistes, inspirés par l'exemple russe, déclenchèrent des grèves et une propagande pacifiste, qui ne fut sans doute pas étrangère aux mutineries de mai. » On retrouve la même rhétorique dans le manuel publié en 1968 chez Bordas [Bouillon, 1968] qui après une présentation très classique de l'offensive Nivelle, en arrive au « Paroxysme de la crise morale : elle fut très grave en France où l'échec de l'offensive d'avril provoqua la mutinerie de plusieurs régiments; la propagande en faveur de la fin des combats se développait, mêlant partisans sincères de la paix et agents "défaitistes" stipendiés de l'Allemagne ; les socialistes rompirent l'union sacrée. Tous les belligérants connurent une recrudescence des grèves. » 
POUR LES MUTINS DE 1917 ?

En revanche, les nouveaux programmes de 1982 semblent induire une véritable rupture et s'accompagnent de la floraison d'une nouvelle série de manuels ${ }^{3}$. La Première Guerre mondiale n'est désormais plus traitée en Terminale, mais à la fin du programme de Première. C'est aussi l'époque à laquelle on assiste à la transposition dans les manuels du secondaire des travaux universitaires sur la Grande Guerre. Or ceux-ci ont connu un net développement dans les années soixante et soixante-dix avec, entre autres, la publication des thèses d'Antoine Prost, Guy Pédroncini ou JeanJacques Becker qui deviennent à leur tour directeurs de collection chez les éditeurs scolaires. On voit apparaître des thèmes nouveaux comme «l'autre front $»^{4}$ ou les femmes... Par ailleurs, la Révolution russe et ses conséquences font dorénavant l'objet de chapitres à part entière, autonome de la Première Guerre mondiale. Enfin, les documents se multiplient occupant souvent la moitié de l'espace au sein des manuels.

Pour les mutineries, le changement vient principalement du fait qu'elles sont dorénavant intégrées dans un contexte plus large et davantage politisé, sans qu'il ne soit plus pour autant besoin de recourir à l'argument de la propagande pacifiste (ou défaitiste). Cette situation est de fait paradoxale : alors même qu'elles sont dépolitisées dans l'analyse savante (la thèse de G. Pédroncini met en avant leur caractère spontané et non organisé politiquement contre les analyses antérieures d'une contagion révolutionnaire), elles en viennent être s'articulées plus étroitement avec un ensemble de protestations à caractère politique (grèves, mouvements pacifistes) dans la construction même du récit de la guerre au sein des manuels scolaires.

C'est le changement de régime narratif qui permet de comprendre cette évolution de traitement. Après une longue période durant laquelle l'histoire chronologique et militaire du conflit dominait, les programmes de 1982, héritier pour partie du

\footnotetext{
${ }^{3}$ Le marché des manuels connaît aussi à cette période une augmentation notable, en lien direct avec celle du nombre des lycéens.

4 Titre d'un numéro spécial du Mouvement social.
} 
renouvellement porté par ceux des années soixante, se construisent désormais essentiellement autour de thématiques (même si la dimension chronologique est loin d'être abandonnée quand il s'agit d'étudier un conflit). Cette nouvelle approche se traduit par le multiplications des doubles pages consacrées aux tranchées, aux civils, à la guerre industrielle ou à la mémoire, dans lesquelles les documents sont très nombreux. Dès lors, les mutineries se trouvent au cœur d'une tension : doivent-elles être intégrées aux approches thématiques ou conserver leur place dans la trame causale de la chronologie? Les manuels du début des années 1980 sont un reflet fidèle de l'attention préférentielle portée à l'époque à une approche en terme d'histoire sociale, même si les mutineries n'en conservent pas moins leur place dans ce qui reste de narration chronologique. Mais la focale portée sur une année 1917, année dont la caractéristique principale est d'être le moment de concentration d'un mouvement de contestation général tend à les inscrire durablement dans la perspective d'un mouvement sinon conjoint, du moins si concomitant qu'il implique des liens étroits entre les différents phénomènes.

Dans le manuel dirigé par Antoine Prost en 1982, une double page de documents est consacrée aux « mécontentements ». Les mutineries font l'objet d'un court extrait (c'est le texte le plus bref de la page), tiré de Poincaré, L'année trouble, 1917, et strictement descriptif. D'autres documents traitent du mécontentement des soldats mais sans jamais faire référence aux mutins (la « Chanson de Craonne» par exemple ou un extrait de Louis Barthas [Barthas, 1978] décrivant les conditions de vie difficiles dans les tranchées, lors des combats de Lorette); et prennent place aux côtés des autres mécontentements. Dans la partie consacrée au cours proprement dit, le second chapitre traite de «La crise de 1917 » avec un long développement sur le pacifisme, Zimmerwald et Kienthal. Les mutineries sont insérées très naturellement dans un contexte de grèves général, et on trouve dans la même phrase mention des fraternisations et des désertions avec un exemple français (Chemin des Dames) et un exemple allemand (Kiel). L'accent est davantage porté sur les conditions qui ont permis la fin de ces mouvements de protestation divers, avec une description de la reprise en main par le gouvernement, une photographie de la visite de Pétain dans les cantonnement complétée par une légende qui indique les mesures prises à la suite des 
mutineries.

Dans le Berstein-Milza de 1982, une double page de cours et de documents est consacrée aux « crises de 1917 ». Les auteurs insistent essentiellement sur les causes socio-économiques (l'hiver des rutabagas) et mentionnent les courants pacifistes. Dans le second paragraphe, consacré à la manifestation des crises, on trouve, en premier lieu, les « mouvements d'indiscipline militaire » auxquels sont dédiés environ 1/3 du paragraphe. Ce «mouvement s'explique par le réflexe de survie d'hommes durement éprouvés par les offensives meurtrières ». Sont également mentionnés Kiel et les désertions qui suivent Caporetto. En ce qui concerne les documents, c'est un court extrait de lettre de soldat tiré du Pédroncini qui a été choisi.

C'est le Bordas qui contient le développement le plus substantiel [Bouillon, 1983] avec de longs passages sur les tranchées et sur l'autre front, accompagnés de nombreux documents (une page de documents pour une page de cours). La crise de l'année 1917 est présentée comme une crise de lassitude, à l'échelle de tous les belligérants, dans un contexte général d'aspiration à la paix. "Toutefois, ses manifestations, multiformes, recouvrent aussi une remise en cause du sens même de la guerre, de ses objectifs. Pourtant, à l'exception de la Russie où la crise débouche sur une révolution et la paix séparée, la poursuite des combats n'est pas remise en cause. La crise militaire s'avère la plus dangereuse. Des mutineries se développent au front. » Après mention des autres pays, une quinzaine de lignes est consacrée aux mutineries françaises avec des données chiffrées. Les responsables désignés sont l'offensive mal préparée et la multiplication des profiteurs de guerre. Après l'intervention de Pétain, « la censure rigoureuse empêche tout relais avec le mécontentement de l'arrière ». S'ensuit une longue description de la crise sociale, qui par son inscription matérielle fait le lien entre les deux phénomènes. En document, un long extrait de Barthas est proposé, celui-là même qui fait mention des observations qu'il a faites du mouvement ; complété par le texte de la Chanson de Craonne, présentée sous le titre de « chanson subversive ».

Pourtant, certains manuels restent relativement imperméables à cette nouvelle lecture «globalisante» et conservent aux mutineries leur spécificité toute militaire. Ainsi, dans le manuel publié par Scodel [Dubreuil, 1982], le paragraphe consacré aux 
mutineries, inséré classiquement dans la partie du cours sur 1917, est totalement déconnecté des conditions socio-économiques, présentées en détail dans le chapitre suivant. Il n'en reste pas moins qu'elles sont l'objet d'un développement substantiel (près du quart de la leçon) et que la contagion révolutionnaire russe est évoquée : « $\mathrm{Ce}$ sanglant échec, joint à la fatigue des hommes et à une certaine propagande pacifiste influencée par les événements de Russie, provoque dans l'armée une vague de mutineries. La moitié des divisions est touchée plus ou moins sérieusement; début juin, un régiment veut même marcher sur Paris où paradent, disent les révoltés, incapables et embusqués. Pétain, qui a remplacé Nivelle le 16 mai, ramène le calme par des mesures d'humanité : permissions élargies, ordinaire amélioré, abandon des offensives coûteuses. Au total, sur 554 condamnations à mort, une quarantaine seulement furent exécutées. » Un extrait d'une lettre de poilu traitant Nivelle de bourreau et de la chanson de Craonne complète le texte du cours. On retrouve presque mot pour mot la même présentation dans le manuel Istra de 1988 [Wagret, 1988].

\section{Conclusion:}

La lecture des manuels de lycées sur une soixantaine d'années ne fait pas apparaître de véritable évolution dans la place et le traitement des mutineries de 1917, la place qui leur est consacrée tendant même à décroître naturellement du fait de l'augmentation générale des thèmes à traiter au cours d'une même année scolaire. On peut cependant remarquer les effets d'un contexte plus général (une lecture plus politique en période de guerre froide, une analyse plus sociale après les années 1970). Reste que les mutineries, si elles sont toujours traités, le sont généralement avec moins de détails que d'autres aspects de la guerre, qu'il s'agisse des batailles dans la première période et des grèves et mouvements pacifistes dans la seconde. D'une certaine façon, l'introduction d'une lecture plus fortement contextuelle (l'année 1917 comme moment de toutes les protestations) a pour effet de rendre leurs causes moins compréhensibles. Dans la mesure où la description de l'offensive de Nivelle devient de plujs en plus succincte, et où elles ne sont jamais mises en regard avec les conditions du combat et de la vie dans les tranchées, leur inscription dans une protestation multiforme tend à masquer leurs spécificités. 
Paradoxalement, l'éloignement temporel ne semble pas avoir permis aux mutineries de gagner leur place dans l'histoire enseignée. Elles pâtissent directement de la diminution des pages consacrées à la Première Guerre mondiale et s'inscrivent dorénavant dans un contexte trop général pour pouvoir faire l'objet d'un traitement particulier. La disparition des descriptions de l'offensive, sensible depuis les programmes du début des années soixante a eu comme effet leur décontextualisation. Celle de Caporetto ou de Kiel ne permet plus leur traitement dans un contexte comparatiste.

AIMOND C.-E. (Mgr) (1949), Histoire contemporaine du milieu du XIXe siècle à 1939, de Gigord, Paris.

ALLYNCK P. (1946), L'époque contemporaine 1848-1939, Masson, Paris.

BARTHAS L. (1978), Les carnets de guerre de Louis Barthas, tonnelier, 1914-1918, Éditions François Maspero, Paris.

BERSTEIN S., MILZA P. (1982), Histoire, Première, Hatier, Paris.

BOUILLON J. (1968), Le monde contemporain, Bordas, Paris.

BOUILLON J., SOHN A.M., BRUNEL F. (1983), Le monde contemporain, Bordas, Paris.

CALVET, ANCEL (1929), Histoire contemporaine depuis le milieu du XIXe siècle, Delagrave, Paris.

CAZALS R., ROUSSEAU F. (2001), 14-18. Le cri d'une génération, Privat, Toulouse. DUBREUIL (1982), Histoire 1914/1939, Scodel, Paris.

GENET L. (1945), L'époque contemporaine (1848-1919), Hatier, Paris.

JARRY E. (1955), L'époque contemporaine, Editions de l'Ecole, Paris.

HANOTAUX G. (1923), Histoire illustrée de la guerre de 1914, Tome 15 (fin juillet 1918-...), Edition française illustrée, Paris.

MALET, ISAAC (1930), Histoire contemporaine depuis le milieu du XIXe siècle, Hachette, Paris.

MERMEIX (1919), Nivelle et Painlevé. La deuxième crise du commandement, Ollendorff, Paris.

MORAZE C., WOLFF P. (1949), L'époque contemporaine (1852-1948), A. Colin, Paris. 
OFFENSTADT N. (2005), Posteface à ROLLAND D., La grève des tranchées, Imago, Soissons.

OLIVERA P. (2001), La politique lettrée. Les essais politiques en France (1919-1932), thèse de doctorat d'histoire, Université de Paris 1.

PAINLEVE P. (1919), « La Vérité sur l'offensive du 16 avril 1917 », La Renaissance politique, littéraire, économique, Paris, $7^{\mathrm{e}}$ année, numéro spécial, novembre.

PROST A. (1982), Histoire Première, A. Colin, Paris.

RENOUVIN P. (1934), La Crise européenne et la Grande guerre (1904-1918), F. Alcan, Paris.

ROULIE (1963), Le Monde contemporain, de Gigord, Paris.

TISON H. (1994), « La mémoire de la guerre 14-18 dans les manuels scolaires français d'histoire (1920-1990) », in Guerre et culture. 1914-1918, A. Colin, Paris.

TISON H. (1995), «L'image du poilu dans les manuels scolaires d'histoire, 19201994 », in ALLAIN J.-C. (dir.), Des étoiles et des croix. Mélanges offerts à Guy Pedroncini, Economica, Paris.

WAGRET (1988), Histoire. Première, Istra, Paris. 